\title{
Guías para el manejo de la oxigenoterapia domiciliaria en pediatría. Parte 2: Sistemas de administración, suspensión del $\mathrm{O}_{2}$. Oxigenoterapia en situaciones especiales
}

Guidelines for home oxygen therapy management.

Part 2: Administration systems, oxygen therapy suspension. Special situations

Comité Nacional de Neumonología, SAP*

http:/ /dx.doi.org/10.5546/aap.2013.549

\section{SISTEMAS DE \\ ADMINISTRACIÓN DE $\mathrm{O}_{2}$}

El $\mathrm{O}_{2}$ debe prescribirse como cualquier otra medicación, bajo criterios de responsabilidad médica. Debe ser siempre $\mathrm{O}_{2}$ medicinal que responde a las normas de bioseguridad contempladas por el ANMAT. ${ }^{16}$ Hay que advertirles a los pacientes sobre los posibles accidentes que pueden ocurrir durante la administración y que deben registrarlos si suceden. En los Anexos 1 y 2 (en páginas electrónicas) se adjuntan los formularios de solicitud de $\mathrm{O}_{2}$.

Existen diferentes sistemas de administración (Tabla 1). a. $\mathrm{O}_{2}$ en cilindro: el $\mathrm{O}_{2}$ gaseoso se presenta en cilindros de distintas capacidades pintados de blanco con una cruz verde en la parte superior. Se almacena en forma gaseosa y se comprime a una presión de 200 bares. Es la forma de administración más antigua y más extendida en la Argentina (Figura 1).

Debido a esta presión, el volumen de $\mathrm{O}_{2}$ gaseoso que pueden liberar los cilindros es importante:

- 6000 litros o $6 \mathrm{~m}^{3}$ para los cilindros grandes.

- 3000 litros o $3 \mathrm{~m}^{3}$ para los cilindros medianos.
Correspondencia:

Dra. Verónica Giubergia: verogiubergia@gmail. com

Dr. Claudio Castaños: ccastanos@garrahan. gov.ar

Fernando Javier Vila: fjvila@hotmail.com

Conflicto de intereses: Ninguno que declarar.

Recibido: 29-5-2013 Aceptado: 30-7-2013
Secretaria del Comité: Dra. Verónica Giubergia Coordinadores: Dres. Fernando Vila y Claudio Castaños Participaron en la discusión y redacción de esta guía los siguientes miembros del Comité de Neumonología: Dres. Giubergia Verónica, Vila Fernando, Aguerre Verónica, Pawluk Víctor, Dalessandro Virginia, Pereyro Silvia, Nadeo Julio, Paba Patricia, Castaños Claudio, Luque Graciela, Moncada Karina, Bonina Ángel, Moro Leonor, Urrutigoiti Jorge, Inwentarz Sandra, DiTulio Nicolás, Álvarez Daniel, Szulman Gabriela, Magadan Corina, Cracovsky Gabriel, Bodas Pablo, Balinotti Juan, Giorgetti Mariano, Smith Silvina, Ginestet María Eugenia, Andreottola María Elena, Rentería Fernando, Abram Lina, Martinchuck Migliazza Gisela, Fraga Marcela, Benítez Araceli, Baratta Sandra, Melillo Karina, Lombardero Lorena, Robles Raúl, Massa Sabrina, Adot Fernando, Cappelino Marcela, Barria Sandra, Castineiras Ana, Fiamingo Alfio, Petti Daniela, Tucci Nadia, Reches Beatriz, Fleitas Hugo, Bisero Elsa, Diego Dagnino, Kruger Anahí, Finochiaro Juan, Olagaray Lucas, Borda Mauricio, Taire Damián, Cipriani Silvina, Bujedo Elizabeth, Moreno Laura, Lepera Cecilia, Parra Luis, Lubovich Silvina, Salim Maxi, Pisapia Néstor, Toloza Rodolfo, Meneghuzzi Alejandra, Gallardo Liliana, Razovich Laura, Bonifachich Elena, Díaz Nora, Pierini Judith, Solís Teresa. 
- 1000 litros o $1 \mathrm{~m}^{3}$ para los cilindros pequeños (de aluminio para transporte).

- 444 litros o 0,5 $\mathrm{m}^{3}$ para cilindros más pequeños (de aluminio para transporte).

Como la presión del gas a la salida del cilindro es muy alta para poder utilizarlo con seguridad, se añade un regulador que la disminuye a 3 bares aproximadamente. Un manómetro permite verificar cuál es la presión de salida. Cuando el cilindro está lleno y abierto, la aguja del manómetro marca de 150 a 200 bares. A medida que este se vacía, la presión va descendiendo lentamente. Un indicador de volumen fijado en el regulador permite la verificación y el ajuste del flujo de $\mathrm{O}_{2}$ (flujímetro).

\section{Ventajas}

- Son independientes de la energía eléctrica; por lo tanto, el sistema no se ve afectado por el corte de corriente.

- Su uso está muy extendido.

- Pueden resultar más económicos cuando el requerimiento de $\mathrm{O}_{2}$ es bajo y el paciente no deambula.

\section{Desventajas}

- Poca autonomía si los requerimientos de $\mathrm{O}_{2}$ son elevados.

- Requieren la renovación frecuente de los cilindros.

- Ocupan mucho espacio para el almacenamiento debido a su volumen.

- Si los requerimientos son altos el costo puede ser mayor que el de otros sistemas.

- Pueden producirse accidentes por la alta presión a la que sale el gas o por el peso de los tubos.
- No es conveniente para los pacientes que deambulan.

- Deben proveerse al menos dos tubos de $6 \mathrm{~m}^{3}$ adecuadamente preparados.

Se recomienda fijar los cilindros, atarlos a la pared para evitar caídas y colocarlos lejos del fuego y de artefactos que irradian calor (cocinas, estufas), ya que el $\mathrm{O}_{2}$ es comburente y pueden explotar. Lo ideal es que al menos estén a 6 metros de estos artefactos. Se debe tener cuidado cuando se cambia el regulador porque puede ser expedido con mucha fuerza si no está bien colocado y producir accidentes.

b. Concentrador de $\mathrm{O}_{2}$ : es un pequeño artefacto sobre ruedas que pesa entre 20 y 30 kilos, y que se conecta a la red eléctrica normal. Es el método ideal para los pacientes que solo requieren OTD nocturna (Figura 2).

\section{FIGURA 1. Cilindros de $\mathrm{O}_{2}$ ultraliviano y tradicional}
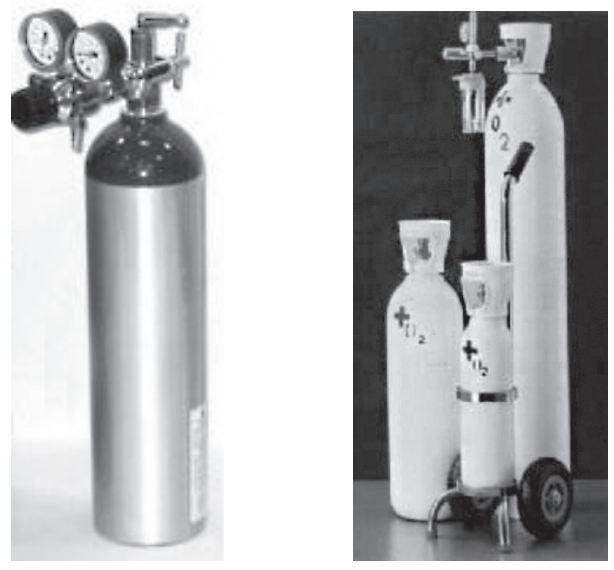

TABla 1. Características de los diferentes sistemas de administración de $\mathrm{O}_{2}$

\begin{tabular}{|c|c|c|c|}
\hline & Cilindros & Concentrador & $\mathrm{O}_{2}$ líquido \\
\hline Dimensión & Tubos de $6 \mathrm{~m}^{3} 130 \times 23 \mathrm{~cm}$ & $38 \times 38 \times 66 \mathrm{~cm}$ & $36,5 \times 77 \mathrm{~cm}$ \\
\hline Peso (kg) & 70 & 28 & 58 \\
\hline Alimentación & Carga por la empresa & Eléctrica $220 \mathrm{~V}$ & Carga por la empresa \\
\hline Flujo de $\mathrm{O}_{2}(\mathrm{~L} / \mathrm{min})$ & 0 a 15 & 0,5 a 8 & 0,25 a 7 \\
\hline Autonomía (a $1 \mathrm{~L} / \mathrm{min}$ ) & 4 días & Indefinido & 15 días \\
\hline Transporte & Tubos de $1 \mathrm{~m}^{3}$ & Tubos de $1 \mathrm{~m}^{3}$ & Mochila de 1,2 L \\
\hline Ventajas & $\begin{array}{l}\text { Vida media larga. } \\
\text { Fácil de conseguir }\end{array}$ & Uso fácil. Móviles & $\begin{array}{l}\text { Baja presión. Mochila de } \\
\text { transporte. Fácil recarga }\end{array}$ \\
\hline Desventajas & $\begin{array}{l}\text { Pesados. Alta presión. } \\
\text { oco volumen de acuerdo con el tamaño }\end{array}$ & $\begin{array}{c}\text { Pesados. Algunos transportables. } \\
\text { Requieren electricidad }\end{array}$ & $\begin{array}{c}\text { Se evapora el } \mathrm{O}_{2} \text {. } \\
\text { Problemas técnicos } \\
\text { (pueden congelarse) }\end{array}$ \\
\hline
\end{tabular}


Funciona a partir del aire ambiente. $\mathrm{El}_{2}$ se separa del nitrógeno por medio de diferentes filtros y luego se concentra, hasta alcanzar una concentración del 95\%. Al funcionar por medio de la corriente eléctrica, se debe solicitar, además, un tubo auxiliar de $\mathrm{O}_{2}$ de 6 $\mathrm{m}^{3}$ para casos de cortes de luz eléctrica y otro de transporte de aluminio de $1 \mathrm{~m}^{3}$.

Ahora se cuenta con concentradores portátiles de batería que pueden ser útiles para los niños. Algunos presentan una válvula inspiratoria que solo se activa con la inspiración profunda del paciente. No son muy apropiados para los niños pequeños, ya que no alcanzan a activarla para que haya flujo de $\mathrm{O}_{2}$. Estos concentradores portátiles pueden ser útiles para los niños que concurren a la escuela (Figura 3).

\section{Ventajas}

- Es una fuente permanente de $\mathrm{O}_{2}$ a domicilio.

- Es de fácil utilización, ya que solo hay que regular el flujo.

- El volumen es menor que con los cilindros de $\mathrm{O}_{2}$.

- El desplazamiento del aparato dentro de la vivienda es fácil gracias a sus ruedas.

- No es necesario el recambio de cilindros; por lo tanto, el costo de su funcionamiento es menor que el del sistema anterior.

\section{Desventajas}

- Depende de la corriente eléctrica (gasto que debe afrontar la familia). Requiere garantías de estabilidad de la corriente eléctrica.

- Entrega flujos limitados (<5 L/min). $\mathrm{La} \mathrm{FiO}_{2}$ es menor cuanto mayor es el flujo. Requiere mantenimiento técnico y cambios de filtros.

- No está indicado para viviendas muy precarias.

c. $\mathrm{O}_{2}$ líquido: se compone de un depósito fijo que se instala en el domicilio del paciente, donde se almacena $\mathrm{O}_{2}$ en forma de $\mathrm{O}_{2}$ líquido y un pequeño depósito de $\mathrm{O}_{2}$ portátil que se llena en el domicilio. Un litro de $\mathrm{O}_{2}$ líquido equivale a 880 litros de $\mathrm{O}_{2}$ gaseoso (Figura 4).

\section{Ventajas}

- No requiere recambios frecuentes ni equipos de auxilio o de transporte.

- Es principalmente útil para los pacientes que usan altos flujos de $\mathrm{O}_{2}$.

- Es el indicado para aquellos que pueden deambular.

\section{FIgURA 3. Concentrador de $\mathrm{O}_{2}$ portátil}

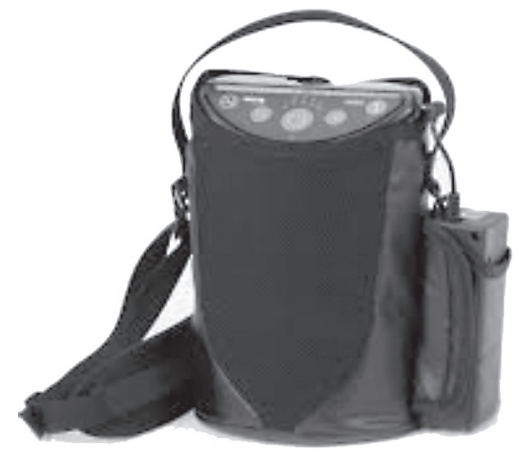

FIgURA 4. Tanques y tubo de transporte de $\mathrm{O}_{2}$ líquido

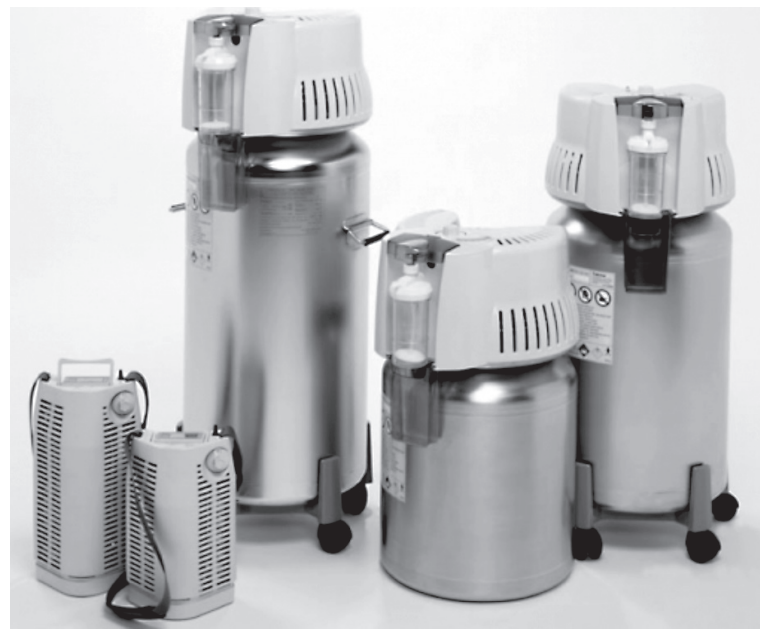




\section{Desventajas}

- Necesita el llenado de la fuente fija por un distribuidor acreditado según el consumo del paciente.

- $\mathrm{El} \mathrm{O}_{2}$ se evapora cuando la fuente de $\mathrm{O}_{2}$ no funciona correctamente.

- Costo elevado.

- Cuando el domicilio es muy distante del proveedor (mayor de 100-150 km) muchos proveedores no pueden aseguran el aporte.

- Puede producir quemaduras por frío si el sistema tiene pérdidas.

\section{Elección del sistema}

No existe ninguna evidencia que muestre que un sistema es mejor que otro con respecto al aporte de $\mathrm{O}_{2}$. La elección dependerá de las características del paciente: la edad, la posibilidad de deambulación, la cantidad de litros de $\mathrm{O}_{2}$ que consume por día, si solo lo requiere de noche o las 24 horas, y de las condiciones socioeconómicas del usuario.

\section{MÉTODOS DE ADMINISTRACIÓN DE $\mathrm{O}_{2}$}

Hay una variedad de sistemas para la administración de $\mathrm{O}_{2}$ en los niños. Estos se clasifican en sistemas de bajo y de alto flujo. Varían en la eficacia, la complejidad, la precisión en la $\mathrm{FiO}_{2}$, el costo y la tolerancia por parte del paciente.

Los sistemas de bajo flujo no cubren las demandas inspiratorias totales del paciente, por lo que la $\mathrm{FiO}_{2}$ obtenida varía en relación con el flujo de $\mathrm{O}_{2}$ administrado, el flujo inspiratorio y el volumen minuto del paciente. Que el sistema sea de bajo flujo no significa necesariamente una $\mathrm{FiO}_{2}$ baja. Puede suministrar una $\mathrm{FiO}_{2}$ baja o alta, pero la $\mathrm{FiO}_{2}$ real solo puede ser estimada porque el paciente está respirando una cantidad de aire ambiente no determinada. Los sistemas de bajo flujo son la cánula nasal, el catéter nasal, la máscara simple y las máscaras con reservorio. Estos sistemas se indican en pacientes con patrón $\mathrm{y}$ frecuencia respiratorios estables.

La cánula nasal es simple, fácil de usar, de bajo costo, bien tolerada, socialmente bien aceptada, y el paciente puede hablar y alimentarse. Es el sistema indicado para el manejo de la oxigenoterapia crónica. ${ }^{2}$

$\mathrm{La} \mathrm{FiO}_{2}$ que aporta varía de acuerdo con los flujos utilizados. Se pueden utilizar flujos de entre 0,5 y $5 \mathrm{~L} / \mathrm{min}$; con estos, la $\mathrm{FiO}_{2}$ alcanzada varía entre 0,22 y 0,44 . Requiere que las fosas nasales estén permeables y produce sequedad nasal con flujos altos. Por este motivo, si el flujo es mayor de $3 \mathrm{~L} / \mathrm{min}$ debe humidificarse y calentarse. Se recomienda que la manguera no supere los 15 metros de largo.

En pacientes especiales que requieran otro sistema de administración, la indicación debe ser evaluada por un pediatra neumólogo.

Las máscaras de traqueostomía y las piezas en T solo están indicadas para la administración de $\mathrm{O}_{2}$ en los pacientes con una vía aérea artificial. Se debe humidificar y filtrar el $\mathrm{O}_{2}$. Los pacientes que tienen traqueostomía deben usar un tubo en T si tienen Bipap, ya que no es aconsejable colocar el $\mathrm{O}_{2}$ directamente en la máscara.

\section{FACTORES POR EVALUAR EN LOS NIÑOS CANDIDATOS A LA OXIGENOTERAPIA DOMICILIARIA}

a. Estabilidad clínica del paciente: mejoría de la enfermedad pulmonar crónica y estabilidad de esta con ausencia de intercurrencias o descompensaciones durante los 10 días previos al egreso hospitalario. Requerimientos de flujos de $\mathrm{O}_{2} \leq 1 \mathrm{~L} / \mathrm{min}, \mathrm{SpO}_{2}$ respirando aire ambiente $\geq 88 \%$ y ganancia ponderal adecuada para la edad.

El paciente debe estar estudiado y deben haberse descartado todas las causas de insuficiencia respiratoria crónica que puedan tratarse. En los niños con enfermedad pulmonar crónica neonatal, es conveniente realizar un ECG o ecocardiograma a fin de descartar HTP.

b. Evaluación familiar: evaluar la estructura familiar, la situación laboral y la vivienda. Esta debe estar en condiciones habitables y, en lo posible, contar con un teléfono de contacto. Se debe entrenar al grupo familiar y deben participar en forma activa en los cuidados del niño antes del alta.

c. Disponibilidad de recursos y su sostén: están relacionados con las posibilidades de ayuda social, asistencia médica y cobertura de los gastos que demanda este tipo de atención.

d. Tabaquismo: debe evaluarse la presencia de fumadores en el domicilio y prohibir el tabaquismo en el hogar y en todos los ambientes en que se encuentre el paciente con OTD.

e. Se debe evaluar la $\mathrm{SpO}_{2}$ en diferentes momentos: durante el sueño, la alimentación y la actividad.

\section{SUSPENSIÓN DEL $\mathrm{O}_{2}$}

Las indicaciones para la suspensión son las siguientes: ${ }^{2}$ 
- $\mathrm{SpO}_{2}$ en aire ambiente $\geq 93 \%$ en diferentes situaciones (llanto, ejercicio, alimentación) con crecimiento adecuado, evolución favorable de la EPC y ecocardiograma sin signos de HTP.

- Requerimiento de $\mathrm{O}_{2} \leq 0,1 \mathrm{~L} / \mathrm{min}$.

- Iniciar la suspensión en forma progresiva durante la vigilia con controles semanales.

- Si a las 4 a 6 semanas continúa con controles de $\mathrm{SaO}_{2} \geq 93 \%$ respirando aire ambiente con todos los parámetros mencionados previamente, se recomienda efectuar $\mathrm{SpO}_{2}$ durante el sueño de al menos 6 horas. Si esta es $\geq 93 \%$ con aire ambiente o la $\mathrm{SpO}_{2}$ es $90 \%$ a $93 \%$ menos del $5 \%$ total del sueño se podrá suspender la OTD y evaluar periódicamente.

- Independientemente de que la $\mathrm{SpO}_{2}$ sea $\geq 93 \%$, si la ganancia de peso se detuvo, manteniendo el mismo aporte nutricional anterior a la suspensión, debe indicarse nuevamente OTD.

- Si la caída es $>90 \%$ no se puede suspender.

- Ni la taquicardia ni la taquipnea por sí solas sirven como indicación de OTD.

Durante los primeros 3 meses de la suspensión, el paciente debe permanecer con el equipo de $\mathrm{O}_{2}$ en su domicilio, ya que podría requerirlo nuevamente en caso de una intercurrencia respiratoria infecciosa.

\section{SEGUIMIENTO}

Es conveniente que el seguimiento de estos pacientes se realice en centros de referencia que puedan realizar estudios de laboratorio completo con gases en sangre, ECG y ecocardiograma; estudios funcionales respiratorios (espirometría, prueba de la marcha) y estudios por imágenes. Si esto no es posible, el paciente debería ser evaluado al menos tres veces por año en centros de referencia para poder vigilar su evolución. ${ }^{2}$

- Es necesario para el seguimiento que los centros de referencia cuenten con un equipo multidisciplinario que incluya pediatra, neumólogo, kinesiólogo y asistente social, además de otras especialidades, como nutricionistas y cardiólogos, o endoscopia respiratoria.

- Se debe incluir en el seguimiento lo concerniente a la cobertura de los gastos, la provisión de los equipos y su mantenimiento.

- Debe asegurarse a los pacientes la provisión de ambulancias para el traslado a los centros de control (que deben tener $\mathrm{O}_{2}$, y atención y traslado en caso de emergencias).
- Los requerimientos de $\mathrm{SpO}_{2}$ domiciliaria deben evaluarse para cada paciente y no es necesario el oxímetro para el seguimiento domiciliario.

- Debe advertirse a los padres sobre posibles complicaciones del uso del $\mathrm{O}_{2}$ (quemaduras, traumatismos) y registrarlas si ocurrieran.

- Debe educarse a la familia sobre las normas de higiene de equipos y sistemas de administración.

\section{OXIGENOTERAPIA EN SITUACIONES ESPECIALES}

a. Oxigenoterapia en la escuela

Debe haber buena comunicación entre el equipo de salud y la escuela. Se debe considerar:

- El equipo de OTD debe ser liviano y fácil de transportar por el niño.

- Es conveniente que haya al menos un tubo de $\mathrm{O}_{2}$ en la escuela y tomar las medidas de seguridad para que no se produzcan accidentes.

- El personal escolar debe estar entrenado en el manejo del equipo y saber a quién contactar ante eventuales problemas.

Existen recomendaciones para padres y para oxigenoterapia en el colegio, disponibles en: http://www.chicos.net/red.

\section{b. Oxigenoterapia en los automóviles}

No existe ningún requisito legal que exija poner etiquetas en las ventanas cuando se lleva $\mathrm{O}_{2}$ médico para uso personal.

Los cilindros deben fijarse de forma segura en el baúl del vehículo o en los asientos traseros, y no deben ser transportados en el asiento delantero. Los cilindros se podrían usar en el transporte público (por ejemplo, colectivos y trenes) siempre y cuando estén en buen estado y se cumplan las medidas de seguridad.

Los concentradores de $\mathrm{O}_{2}$ portátiles que funcionan con una batería se pueden enchufar en el encendedor del coche.

\section{c. Oxigenoterapia en los vuelos}

Los aviones de línea con la cabina presurizada a una altura de 1500 a 2000 metros tienen una $\mathrm{FiO}_{2}$ entre $15 \%$ y $17 \%$ y no deben ser un problema para las personas sin requerimientos de $\mathrm{O}_{2}$ suplementario.

Si bien la gran mayoría de los lactantes y niños sanos no deberían presentar niveles significativos de hipoxemia durante los vuelos, aquellos con enfermedad respiratoria pueden encontrarse en riesgo de sufrir eventos hipóxicos. 
Lamentablemente, las pruebas de función pulmonar habituales, tanto como la $\mathrm{SpO}_{2}$ en aire ambiente, no fueron buenos predictores de la aparición de hipoxemia durante el vuelo.

\section{Métodos para determinar el riesgo de hipoxemia en la altura}

El método más difundido para simular el ambiente de vuelo y evaluar sus repercusiones es la prueba de simulación de hipoxia en altura (HAST), que permite determinar el requerimiento de $\mathrm{O}_{2}$ suplementario. ${ }^{17-18}$

En los niños con requerimientos de $\mathrm{O}_{2}$ en los que no se pueda realizar esta prueba, se recomienda duplicar los flujos de $\mathrm{O}_{2}$ durante el vuelo.

Se ha publicado escasa evidencia que defina con exactitud las indicaciones para la realización del HAST prevuelo en los niños. Las guías publicadas sugieren:

- En los RN de término esperar una semana después de alcanzar el término antes de viajar para asegurarse de que el niño es sano.

- Los lactantes prematuros con o sin DBP que no alcanzaron el término deberían contar con disponibilidad de $\mathrm{O}_{2}$ en el vuelo y recibirlo a 1-2 L/min en caso de presentar síntomas o de disminución de la $\mathrm{SpO}_{2}$ en vuelo $<85 \%$.

- Los pacientes con OTD a nivel del mar, deberían duplicar el flujo de $\mathrm{O}_{2}$ durante el vuelo.

- Los pacientes con enfermedades restrictivas graves de cualquier causa se encuentran en riesgo de presentar hipoxemia durante el vuelo.

- El HAST tiene indicación en los lactantes con historia de DBP sin requerimientos de $\mathrm{O}_{2}$, en los niños que han suspendido el aporte de $\mathrm{O}_{2}$ suplementario en los 6 meses previos al vuelo, en los niños con FQ u otras enfermedades pulmonares obstructivas crónicas con volumen espiratorio forzado en el primer segundo (VEF1) < 50\% y en los niños con enfermedad restrictiva grave (enfermedades intersticiales, escoliosis graves, distrofias torácicas, enfermedades neuromusculares).

Los niños menores de un año con una $\mathrm{SpO}_{2}$ $<85 \%$ o mayores de un año con una $\mathrm{SpO}_{2}$ de $90 \%$ durante el HAST deberían contar con $\mathrm{O}_{2}$ suplementario disponible durante el vuelo.

- Los niños que han presentado un neumotórax o bullas hipertensivas deben esperar una semana luego de la resolución del cuadro o 14 días en caso de haber sido traumático.
Para los pacientes que deben viajar en avión hay que tener en cuenta algunas consideraciones:

- No todas las aerolíneas tienen posibilidad de llevar a pacientes con $\mathrm{O}_{2^{\prime}}$ por lo cual esto debe arreglarse previamente, con un tiempo mínimo de al menos 48 horas. Es aconsejable el transporte hasta el avión con ambulancia y que también espere una ambulancia en el destino.

- Es aconsejable que el paciente lleve una lista con toda la medicación que recibe refrendada por su médico de cabecera.

- Son preferibles los vuelos directos. Si no es posible y deben realizarse conexiones, hay que arreglar previamente la provisión de $\mathrm{O}_{2}$ en el aeropuerto.

- Durante el vuelo el paciente no debe usar su propio $\mathrm{O}_{2}$ Debe ser provisto por la línea aérea.

- Durante el vuelo los flujos de $\mathrm{O}_{2}$ están limitados en la mayoría de las líneas aéreas a 2 y 4 L/min; el médico de cabecera debe elegir el flujo indicado.

\section{BIBLIOGRAFÍA}

1. Neff TA, Petty TL. Long-term continuous oxygen therapy in chronic airway obstrucción: mortality in relationship to cor pulmonale, hypoxia and hypercapnia. Ann Intern Med 1970;72:621-6.

2. Balfour-Lynn IM, Field DJ, Gringras P, Hicks B, Jardine E, et al. On behalf of the Paediatric Section of the Home Oxygen Guideline Development Group of the BTSStandards of Care Committee.BTS guidelines for home oxygen in children. Thorax 2009;64 (Suppl II):1-26.

3. Urschitz NS, Wolff J, Sokolik C, Eggebrecht E, et al. Nocturnal arterial oxygen saturation and academic perfomance in a comunity sample of children. Pediatrics 2005;115:e204-9.

4. Askie LM, Henderson-Smart DJ, Irwig L, Simpson JM. Oxygen saturation targets and outcomes in extremely preterm infants. N Eng J Med 2003;349:959-67.

5. The STOP-ROP Multicenter Study Group. Supplemental Therapeutic Oxygen for Prethreshold Retinopathy Of Prematurity (STOP-ROP), a randomized controlled Trial. I: primary outcomes. Pediatrics 2000;105:295-310.

6. Weinberger B, Laskin DL, Heck DE, Laskin JD. Oxygen toxicity in premature infants. Toxicol Appl Pharmacol 2002;181:60-7.

7. Crockett A, Cranston J, Antic N. Domiciliary oxygen for interstitial lung disease. Cochrane Database Syst Rev 2001;3:CD002883.

8. Murtagh P, Giubergia V, Viale D, Bauer G, Pena HG. Lower respiratory infections by adenovirus in children. Clinical features and risk factors for bronchiolitis obliterans and mortality. Pediatr Pulmonol 2009;44(5):450-6.

9. Zinman R, Corey M, Coates AL, Canny GJ, et al. Nocturnal home oxygen in the treatment of hypoxemic cystic fibrosis patients. J Pediatr 1989;114:368-77.

10. Aljadeff G, Gozal D, Bailey-Wahl SL, Burrel B. Effects of overnight supplemental oxygen in obstructive sleep apnea in children. Am J Respir Crit Care Med 1996;153:51-5.

11. Widlitz A, Barst RJ. Pulmonary arterial hypertension in children. Eur Respir J 2003;21:155-76. 
12. Bruera E, de Stoutz N, Velasco-Leiva A, Schoeller T, et al. Effects of oxygen on dyspnoea in hypoxaemic terminalcancer patients. Lancet 1993;342:13-4.

13. Uronis HE, Currow DC, McCrory DC, Samsa GP, et al. Oxygen for relief of dyspnoea in mildly-or non-hypoxaemic patients with cancer: a systematic review and meta-analysis. Br J Cancer 2008;98:294-9.

14. MacLean JE, Fitzgerald DA. A rational approach to home oxygen use in infants and children. Paediatr Resp Rev 2006;7:215-22.
15. Balfour-Lynn IM, Primhak RA, Shaw BNJ. Home oxygen for children: who, how and when? Thorax 2005;60:76-81.

16. ANMAT. [Consulta: 10 de abril de 2013]. Disponible en http//: www.anmat.gov.ar.

17. Thoracic Society Standards of Care Committee Managing passengers with respiratory disease planning air travel: British Thoracic Society recommendations. Thorax 2011;66:130.

18. AguerreV.Viajeen avión, riesgos y previsiones a considerar en los niños. Arch Argent Pediatr 2012;110(1):66-9. 


\section{Anexo 1. \\ Formulario de solicitud de la Oxigenoterapia. Datos clínicos}

Nombre y apellido:

DNI:

$\mathrm{HC}$ N:

Fecha de nacimiento:.......................

Examen clínico:

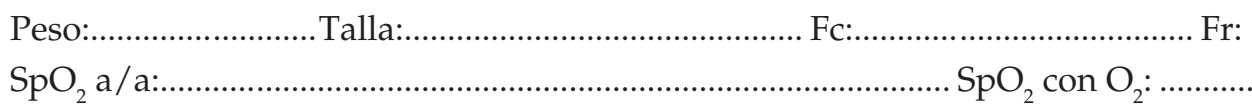

\section{Laboratorio:}

Hemograma:

Urea ..................................... Ionograma:............................... Albúmina:

Gases en sangre:

Rx de tórax:.

Ecocardiograma para valorar HTTP

TAC de tórax*:

Prueba de marcha (caminata de los 6 minutos) *

Espirometría*:

CVF:

VEF1:

FEF 25/75:

Diagnóstico definitivo:

* (si corresponde) 


\section{Anexo 2. \\ Formulario de prescripción del $\mathrm{O}_{2}$}

Nombre y apellido:

$\mathrm{HC} \mathrm{N}$ :.

Domicilio:

Teléfono:

Fecha de ingreso al programa (inicio de seguimiento):

Diagnóstico:

Requerimiento: $\mathrm{L} / \mathrm{min}$

Permanente:

Intermitente:

Indicación:

\section{FUENTE DE ADMINISTRACIÓN:}

1. Cilindros ( 2 tubos de $\mathrm{O}_{2}$ de $6 \mathrm{~m}^{3}=6000 \mathrm{~L}$, con reductor para tubo de $\mathrm{O}_{2}$ con tomagoma, reloj indicador de capacidad del tubo 0 a $300 \mathrm{~kg} / \mathrm{cm}^{3}$ y reloj indicador de salida de flujo reglado de 0 a 3 litros). 1 tubo de $\mathrm{O}_{2}$ de $1 \mathrm{~m}^{3}$ de aluminio ultraliviano $1000 \mathrm{~L}$ para transporte con recarga.

2. Concentrador de $\mathrm{O}_{2}$ eléctrico con servicio técnico garantizado y recambio de filtro, estabilizador de voltaje, tubo de $\mathrm{O}_{2}$ de $6 \mathrm{~m}^{3}=6000 \mathrm{~L}$ (de seguridad) y tubo de $\mathrm{O}_{2}$ de $1 \mathrm{~m}^{3}$ de aluminio ultraliviano 1000 L para transporte con recarga.

3. Tanque de $\mathrm{O}_{2}$ líquido de 32 litros (tipo freelox) con reservorio portátil con capacidad de 1,2 litros.

- No requiere saturómetro permanente en el domicilio del paciente.

- Traslado del paciente y acompañante en ambulancia con $\mathrm{O}_{2}$ a controles programados o consultas de emergencia al hospital.

- Horas de consumo al día: horas.

- Nombre y apellido del médico tratante:

- Hospital o Servicio tratante:

Médico tratante

Jefe de Servicio

Director del Hospital 